\title{
Perceptions of Vietnamese EFL High School Teachers and Students towards English as a Lingua Franca
}

\author{
Nguyen Thien-Bao ${ }^{1 *}$, Yi-Hsuan Gloria Lo $^{2}$
}

${ }^{1}$ National Chung Cheng University, Chiayi County, Taiwan

${ }^{2}$ National Taiwan University of Science and Technology, Taipei, Taiwan

"Corresponding author's email: nguyenthienbao1611@gmail.com

Dhttps://orcid.org/0000-0002-2412-0547

do) https://doi.org/10.54855/ijte.222120

Received: 28/01/2022 Revision: 18/02/2022 Accepted: 22/02/2022 Online: 24/02/2022

ABSTRACT

The purpose of this study is to identify the perceptions of Vietnamese EFL in-service teachers and students from gifted versus non-gifted high schools towards English as a lingua franca (ELF). In this quantitative study, a questionnaire was utilized to collect data that was then analyzed using descriptive and inferential statistics. The results show that both Vietnamese high school EFL teachers and students had positive perceptions towards ELF in general and towards each of the dimensions of ELF in particular. Additionally, there were some statistically significant differences between the perceptions of sub-groups of Vietnamese EFL teachers and students

Keywords: English as a lingua franca (ELF), gifted and non-gifted high schools, high school EFL teachers and students, perceptions. from gifted versus non-gifted high schools. The study contributes to the literature of the field and assists policymakers when it comes to formulating policy to enhance English communication skills at the high school level, particularly in the Vietnamese context. In addition, the current study is beneficial for teachers and learners to have an insight into how they should teach and learn English to communicate effectively.

\section{Introduction}

Globalization has given rise to changes in a myriad of life's facets. The phenomenon also popularizes English as a medium of communication worldwide. This means that the number of non-native English speakers outweighs that of native English speakers. The fact that people interact with partners from various linguistic and cultural backgrounds rather than English is prominent (Curran \& Chern, 2017). Teaching English as a foreign language (EFL), to satisfy the world's new requirements for employees to be fluent in communicative English, has also driven the focus from teaching to test to teaching to communicate. The conventional notion of teaching standard English pronunciation (American English or British English) as well as the idea of using only English in the classroom has no longer been considered dominant. English, in this case, is used as a medium of communication between people who come from different first language backgrounds, which is so-called English as a lingua franca (ELF) (Jenkins, 2012) 
In recent decades, a dramatic increase in the ELF interest has been internationally extended thanks to the preliminary works done by Jenkins (2000) and Seidlhofer (2001). Researchers have also driven their attention to ELF in the Vietnamese context since the first decade of the new millennium. Moreover, one of the important missions of the Vietnamese National Foreign Languages Project is to prepare teachers and learners with sufficient occupational language skills, especially in the fields with free labor mobility within the Association of Southeast Asian Nations economic community (Ministry of Education of Vietnam, 2018). Apparently, English speaking competencies tested at schools may not satisfy the real needs of learners anymore (Butler, Lee \& Peng, 2020). The urgent demand for these students to be able to communicate efficiently with non-native English speakers by using English has been officially recognized and put forward by the authority.

In addition, the jargon 'gifted students' or 'specialized students' have long been utilized to refer to students who achieve excellent academic results in specialized schools (or schools for the gifted) in Vietnam, which 'are established at upper secondary education level for pupils with outstanding achievements in learning to develop their aptitudes for certain subjects while assuring comprehensive general education.' (Vietnamese educational law, Art. 62, 2005). Every year, a gifted high school admits not more than $0.1 \%$ of the total population of the whole province (Decision 82, Art. 3, 2010). To be admitted to a high school for the gifted, students must take the 4-subject examination, including Maths, Vietnamese Literature, English, and the major. The minimum passing score for the major is set at 6.0/ 10.0. Moreover, while studying at a high school for the gifted, students who get the major Grade Point Average (GPA) of the total GPA lower than 6.5, or any other subjects lower than 5.0, will be expelled from the school. Obviously, with the pressure these students have to face, their perceptions are definitely different from those of their regular peers.

Therefore, exploring gifted versus non-gifted teachers' and students' perceptions of these changes is important. The present study contributes to the current literature of ELF with a more comprehensive understanding of high school teachers' and students' perceptions. Also, policymakers may consider the results of this study when framing policy for teaching English communication skills at the high school level. With this study, high school EFL learners and teachers are also more aware of how to teach and learn English so that learners can use English efficaciously in their future jobs.

\section{Literature review}

\section{English as a lingua franca}

For thousands of years, people who do not speak the same native language have longed for a language of communication, a lingua franca (Haberland, 2011). Ruth (2018) defines various use in ELF communication as the difference in using language forms and functions from the conventionalized standard language by English native speakers. EFL research also focuses on investigating the "process of variation itself, on what motivates the variable use of linguistic 
resources in the achievement of communicative purposes in different contexts of use' (Widdowson, 2015, p. 363). As Ruth (2018) states, a reconsideration of established concepts, such as the role and usefulness of norms and standards (Dewey, 2012; Widdowson, 2012), or the usefulness of linguistic categorization for actual language use (Osimk-Teasdale, 2015) based on the research on the way linguistic resources are adapted in ELF contexts is required.

\section{Relevant research on ELF and gifted students}

Although some of the key issues of ELF had been dealt with for decades, research into ELF has emerged only relatively recently (Shohamy \& Hornberger, 2017). According to Kontra and Csizer (2011), the focus of ELF research is on two main aspects. The first targeted feature is the investigation of ELF's characteristics and use (Wu, Mauranen \& Lei, 2020; Pang, 2020; Víctor \& Lanteigne, 2020; Guohai \& Geling, 2021). The second one is the exploration of the perceptions of language teachers and learners towards ELF (Yalçın, Bayyurt \& Alahdab, 2020; Laitinen, 2020; Ambele \& Boonsuk, 2020; Kim, 2021).

In the context of Vietnam, researchers have also paid attention to the phenomenon of ELF (Do, 2000; Ton \& Pham, 2010; Tran \& Moore, 2015; Ho \& Nguyen, 2019; Yao, Garcia \& Collins, 2019; Nguyen, 2019; Phan, 2020), especially on the attitudes of teachers and learners at tertiary level, though the number of studies is small. However, gifted students' perceptions have not received much attention from researchers. From the literature review, only a few of such studies can be found (Vu, 2011; Vu \& Vu, 2012; Nguyen, Jin \& Gross, 2013; Nguyen \& Ngo, 2018). $\mathrm{Vu}$ (2011) examined gifted students' profiles and their perceptions towards a gifted program. $\mathrm{Vu}$ and $\mathrm{Vu}$ (2012) explored how gifted students recognized the effectiveness of the gifted program. Nguyen, Jin, and Gross (2013) investigated the Confucian values in Vietnamese gifted adolescents and their regular peers. Nguyen and Ngo (2018) conducted research on teachers' and students' perceptions towards English as a medium of instruction at a gifted high school in Central Vietnam. Apparently, a study on gifted students' perceptions of ELF would be a great contribution to the gap in the literature and the practical implementation of English language teaching at Vietnamese gifted high schools.

Research Gaps

Apparently, most of the studies conducted on the perceptions towards ELF focus on teachers and students at tertiary level or pre-service teachers. Hardly can studies that focus on identifying the perceptions of in-service high school EFL teachers gifted and non-gifted students towards ELF be found on search engines worldwide, let alone in Vietnam. As a result, this study probes the identification of gifted versus non-gifted high school in-service ELF teachers' and students' perceptions towards ELF.

\section{Research Questions}

Three research questions were addressed in this study:

1. How do Vietnamese high school EFL teachers perceive ELF? Are there any significant differences between EFL teachers' perceptions from gifted versus non-gifted high 
schools towards ELF?

2. How do Vietnamese high school EFL students perceive ELF? Are there any significant differences between the perceptions of gifted versus non-gifted high school EFL students towards ELF?

3. Are there any significant differences between the perceptions of high school EFL teachers and students towards ELF?

\section{Methods}

\section{Pedagogical Setting \& Participants}

Vietnamese EFL in-service high school teachers and students from different high schools were the target subjects of this study. These participants partook in this study voluntarily, and their identities were kept confidential.

The Snowball sampling method was employed in this study. Finally, 125 teachers of English at high schools in Vietnam and 151 Vietnamese high school EFL students were recruited for the pilot study to examine whether the scale (Curran \& Chern, 2017) still maintain reliability and validity when used for new samples. Next, 155 teachers (30 from gifted high schools and 125 from non-gifted high schools) and 300 students (141 from gifted high schools and 159 from non-gifted high schools) who had not participated in the pilot study were recruited for the main study.

\section{Design of the Study}

The current study employed a quantitative research approach. The 20-item scale to measure pre-service teachers' perceptions of ELF developed by Curran and Chern (2017) was adapted. However, with new samples of Vietnamese high school EFL teachers and students, the scale needed piloting.

125 teachers of English at high schools in Vietnam and 151 Vietnamese high school EFL students were recruited for the pilot study. The results showed that the Cronbach's alpha of the scale for teachers and students were .33 and .49 , respectively, which was not satisfactory (Devellis, 2012). Therefore, some modifications were made. Firstly, there is a high probability that the cross-cultural measurement equivalence and construct validity of mixed-worded Likert format scales are challenged (Wong, Rindfleisch, \& Burroughs, 2003); also, Sonderen, Sanderman and Coyne (2013) stated that reverse-worded items both did not help reduce response bias and even contaminated the data scores due to respondents' inattention and confusion. Secondly, through expert validity, one item was reported as 'confusing' since the phrase 'to be proficient speakers of English' could be understood in different ways depending on the background knowledge of different people. Hence, these items were deleted.

The Cronbach's alpha coefficient of the scale after modification was .80 for teachers and .71 for students, indicating that the 11-item scale was reliable for use in the new context (Appendix 1). 
Table 1 shows the newly modified scale's dimensions and its corresponding items.

Table 1

Dimensions and items of the newly modified scale

\begin{tabular}{cll}
\hline Codes & \multicolumn{1}{c}{ Dimensions } & Items \\
\hline D1 & Models of English used in teaching materials & $1,2,3$ \\
D2 & Using English for communication & 4,5 \\
D3 & The role of language and culture in the English classroom & $6,7,8,9$ \\
D4 & Language use in the classroom & 10,11 \\
\hline
\end{tabular}

\section{Data collection \& analysis}

An online questionnaire was both randomly sent via email and posted on Facebook groups for Vietnamese EFL teachers and students.

This quantitative study manipulated SPSS statistical package 23. Descriptive statistics was operated to investigate the perceptions of high school EFL teachers and students' perceptions towards ELF. When the mean scores were higher than 3.5, they were classified as positive. When the mean scores were lower than 2.5 , they were classified as negative. When the mean scores were from 2.5 to 3.5 , they were classified as neutral. Also, an independent-samples $t$-test was used to identify whether the significant differences between perceptions of sub-groups towards ELF existed.

\section{Findings}

Research Question 1. How do Vietnamese high school EFL teachers perceive ELF? Are there any significant differences between EFL teachers' perceptions from gifted versus non-gifted high schools towards ELF?

Generally speaking, as shown in Table 2, the participants possessed a positive perception towards four dimensions of $\mathrm{ELF}(\mathrm{D} 1: \mathrm{M}=3.63, \mathrm{SD}=.79 ; \mathrm{D} 2: \mathrm{M}=3.67, \mathrm{SD}=.71 ; \mathrm{D} 3: \mathrm{M}=$ $4.20, \mathrm{SD}=.57 ; \mathrm{D} 4: \mathrm{M}=3.73, \mathrm{SD}=.75)$ and towards $\mathrm{ELF}$ as a whole $(\mathrm{M}=3.86, \mathrm{SD}=.43)$.

\section{Table 2}

Vietnamese high school EFL teachers' perceptions towards ELF

\begin{tabular}{lll}
\hline Dimensions & $\begin{array}{c}\text { Vietnamese high school teachers }(\mathrm{N}=155) \\
\text { Mean }\end{array}$ & SD \\
\hline Overall & 3.86 & .43 \\
D1 & 3.63 & .79 \\
D2 & 3.67 & .71 \\
D3 & 4.20 & .57 \\
D4 & 3.73 & .75 \\
\hline
\end{tabular}

Table 3 presents Vietnamese high school EFL teachers' perceptions of ELF in more detail. A closer look at Table 3 illustrates that although these teachers believed the utmost importance of an English program was to prepare students for social interactions (Item 4: $\mathrm{M}=4.59, \mathrm{SD}=.61$ ), they seemed to have a negative perception towards whether their students should be mainly 
prepared to communicate with non-native English speakers (Item 5: $\mathrm{M}=2.75, \mathrm{SD}=1.24$ ). Another notable figure is Item $3(\mathrm{M}=3.16, \mathrm{SD}=1.15)$, which presents a rather neutral perceptions of Vietnamese high school EFL teachers towards models of English used in teaching materials by both L1 and L2 speakers. Interestingly, all the items in D3 are among the highest-rated scores of all (Item $6: \mathrm{M}=4.27, \mathrm{SD}=.80 ;$ Item $7: \mathrm{M}=4.39, \mathrm{SD}=.62 ;$ Item $8: \mathrm{M}$ $=4.06, \mathrm{SD}=.90 ;$ Item $9: \mathrm{M}=4.07, \mathrm{SD}=.84$ ).

\section{Table 3}

Perceptions of Vietnamese high school EFL teachers towards ELF in more detail

\begin{tabular}{ccc}
\hline Items & $\begin{array}{c}\text { Vietnamese high school teachers }(\mathrm{N}=155) \\
\text { Mean }\end{array}$ \\
\hline 1 & 4.00 & $\mathrm{SD}$ \\
2 & 3.72 & .94 \\
3 & 3.16 & 1.09 \\
4 & 4.59 & 1.15 \\
5 & 2.75 & .61 \\
6 & 4.27 & 1.24 \\
7 & 4.39 & .80 \\
8 & 4.06 & .62 \\
9 & 4.07 & .90 \\
10 & 3.69 & .84 \\
11 & 3.79 & .98 \\
Overall & 3.86 & .93 \\
\hline
\end{tabular}

An independent samples $t$-test was also conducted (Table 4). The results show that there was a significant difference in perceptions towards D1 between Vietnamese EFL teachers from gifted $(\mathrm{M}=3.93, \mathrm{SD}=0.88)$ and non-gifted high schools $(\mathrm{M}=3.55, \mathrm{SD}=0.76)$ conditions; $\mathrm{t}(153)=$ $2.41, \mathrm{p}=.02$.

\section{Table 4}

Independent samples t-test analysis of the perceptions of Vietnamese teachers from gifted versus non-gifted high schools towards ELF

\begin{tabular}{lcccccc}
\hline Constructs & $\begin{array}{c}\text { Teachers from gifted high } \\
\text { schools } \\
\end{array}$ & \multicolumn{2}{c}{$\begin{array}{c}\text { Teachers from non-gifted } \\
\text { high schools } \\
\end{array}$} & Mean & SD & \multicolumn{2}{c}{$\begin{array}{c}\text { Mean }=125) \\
\text { SD }\end{array}$} & t-value & Sig. \\
\hline Overall & 4.00 & 0.49 & 3.83 & 0.41 & 1.96 & .051 \\
D1 & 3.93 & .88 & 3.55 & .76 & 2.41 & $.02 *$ \\
D2 & 3.73 & .81 & 3.66 & .69 & .51 & .62 \\
D3 & 4.31 & .49 & 4.17 & .59 & 1.12 & .25 \\
D4 & 3.75 & .80 & 3.73 & .75 & .09 & .93 \\
\hline
\end{tabular}

To investigate which items create this statistically significant difference, an independent samples $t$-test analysis was conducted for Items 1,2 , and 3 . Table 5 presents the independent samples t-test analysis of the perceptions of Vietnamese teachers from gifted versus non-gifted high schools towards models of English used in teaching materials. The result shows there was significant difference in Item 3 between Vietnamese EFL teachers from gifted $(\mathrm{M}=4.23$, SD $=.94)$ and non-gifted high schools $(\mathrm{M}=3.90, \mathrm{SD}=.94)$ conditions; $\mathrm{t}(153)=2.55, \mathrm{p}=.01$. 


\section{Table 5}

Independent samples t-test analysis of the perceptions of Vietnamese teachers from gifted versus non-gifted high schools towards models of English used in teaching materials

\begin{tabular}{lcccccc}
\hline Items & $\begin{array}{c}\text { Teachers from gifted high schools } \\
(\mathrm{N}=30)\end{array}$ & \multicolumn{5}{c}{ Teachers from non-gifted high schools } \\
& Mean & $\mathrm{SD}$ & Mean & SD & t-value & Sig. \\
\hline 1 & 4.23 & .94 & 3.90 & .94 & 1.52 & .13 \\
2 & 3.63 & 1.10 & 3.05 & 1.14 & 1.22 & .22 \\
3 & 4.23 & .94 & 3.90 & .94 & 2.55 & $.01^{*}$ \\
\hline
\end{tabular}

Research Question 2. How do Vietnamese high school EFL students perceive ELF? Are there any significant differences between the perceptions of gifted versus non-gifted high school EFL students towards ELF?

In general, as shown in Table 6, the participants possessed a positive perception towards four dimensions of $\operatorname{ELF}(\mathrm{D} 1: \mathrm{M}=3.49, \mathrm{SD}=.79 ; \mathrm{D} 2: \mathrm{M}=3.84, \mathrm{SD}=.73 ; \mathrm{D} 3: \mathrm{M}=4.08, \mathrm{SD}=.63$; $\mathrm{D} 4: \mathrm{M}=3.79, \mathrm{SD}=.93)$ and towards ELF as a whole $(\mathrm{M}=3.82, \mathrm{SD}=.52)$.

\section{Table 6}

Perceptions of Vietnamese high school EFL students towards ELF

\begin{tabular}{ccc}
\hline Dimensions & \multicolumn{2}{c}{$\begin{array}{c}\text { Vietnamese high school students }(\mathrm{N}=300) \\
\text { Mean }\end{array}$} \\
\hline Overall & 3.82 & .52 \\
D1 & 3.47 & .79 \\
D2 & 3.84 & .73 \\
D3 & 4.08 & .63 \\
D4 & 3.79 & .93 \\
\hline
\end{tabular}

Table 7 presents the mean and SD of Vietnamese high school students' perceptions towards ELF in more detail. The most notable feature for the first three items is that English spoken by a range of native speakers seemed to be most favored by these students (Item 1: $\mathrm{M}=3.74$, SD $=1.04)$. Besides, like the teachers, although the students believed the utmost importance of an English program was to prepare students for social interactions (Item 4: $\mathrm{M}=4.54, \mathrm{SD}=.69$ ), they seemed to have a rather neutral perception towards whether their students should be mainly prepared to communicate with non-native English speakers (Item 5: $\mathrm{M}=3.13, \mathrm{SD}=1.20$ ). Another considerable feature is the two second-highest-rated items belonging to D3 that indicate the student's interest in sharing their own cultural values and learning about intercultural communication in the classroom (Item 6: $\mathrm{M}=4.38, \mathrm{SD}=.82$; Item $7: \mathrm{M}=4.29$, $\mathrm{SD}=.79)$. 


\section{Table 7}

Perceptions of Vietnamese high school students towards ELF in more detail

\begin{tabular}{ccc}
\hline Items & \multicolumn{2}{c}{ Vietnamese high school students $(\mathrm{N}=300)$} \\
& Mean & SD \\
\hline 1 & 3.73 & 1.04 \\
2 & 3.51 & 1.11 \\
3 & 3.17 & 1.22 \\
4 & 4.54 & .69 \\
5 & 3.13 & 1.20 \\
6 & 4.38 & .82 \\
7 & 4.29 & .79 \\
8 & 3.68 & 1.04 \\
9 & 3.95 & .92 \\
10 & 3.77 & 1.15 \\
11 & 3.81 & 1.00 \\
Overall & 3.82 & .52 \\
\hline
\end{tabular}

An independent samples $t$-test was also conducted (Table 8). The results show that there was a significant difference in perceptions towards D2 between Vietnamese gifted high school EFL students $(\mathrm{M}=3.74, \mathrm{SD}=.69)$ and Vietnamese non-gifted high school EFL students $(\mathrm{M}=3.93$, $\mathrm{SD}=.76)$ conditions; $\mathrm{t}(298)=-2.26, \mathrm{p}=.024$.

\section{Table 8}

Independent samples t-test analysis of the perceptions of students from gifted versus nongifted high schools towards ELF

\begin{tabular}{|c|c|c|c|c|c|c|}
\hline \multirow[t]{2}{*}{ Dimensions } & \multicolumn{2}{|c|}{$\begin{array}{l}\text { Students from gifted high } \\
\text { schools } \\
(\mathrm{N}=141)\end{array}$} & \multicolumn{2}{|c|}{$\begin{array}{l}\text { Students from non-gifted high } \\
\text { schools } \\
(\mathrm{N}=159)\end{array}$} & \multirow{2}{*}{$\begin{array}{c}\mathrm{t}- \\
\text { value }\end{array}$} & \multirow[t]{2}{*}{ Sig. } \\
\hline & Mean & SD & Mean & SD & & \\
\hline Overall & 3.80 & .47 & 3.83 & .56 & -.40 & .69 \\
\hline D1 & 3.46 & .78 & 3.48 & .81 & -.16 & .87 \\
\hline D2 & 3.74 & .69 & 3.93 & .76 & -2.26 & $.024 *$ \\
\hline D3 & 4.13 & .54 & 4.03 & .70 & 1.31 & .19 \\
\hline D4 & 3.73 & .92 & 3.84 & .95 & -1.01 & .31 \\
\hline
\end{tabular}

An independent samples $t$-test analysis was conducted for Items 4 and 5. Table 9 presents the independent samples t-test analysis of the perceptions of students from gifted versus non-gifted high schools towards using English for communication. The result shows there was significant difference in Item 5 between Vietnamese gifted high school EFL students $(M=2.96, S D=1.15)$ and Vietnamese non-gifted EFL high school students $(\mathrm{M}=3.29, \mathrm{SD}=1.23)$ conditions; $\mathrm{t}(298)$ $=-2.40, \mathrm{p}=.017$. 


\section{Table 9}

Independent samples t-test analysis of the perceptions of students from gifted versus nongifted high schools towards using English for communication

\begin{tabular}{ccccccc}
\hline Items & $\begin{array}{c}\text { Students from gifted high schools } \\
(\mathrm{N}=141)\end{array}$ & \multicolumn{2}{c}{$\begin{array}{c}\text { Students from non-gifted high schools } \\
(\mathrm{N}=159)\end{array}$} \\
& Mean & $\mathrm{SD}$ & Mean & $\mathrm{SD}$ & t-value & Sig. \\
\hline 4 & 4.52 & .70 & 4.57 & .67 & -.61 & .54 \\
\hline 5 & 2.96 & 1.15 & 3.29 & 1.23 & -2.40 & $.017^{*}$ \\
\hline
\end{tabular}

Research Question 3. Are there any significant differences between the perceptions of high school EFL teachers and students towards ELF?

An independent samples t-test was conducted to compare perceptions towards English as a lingua franca between Vietnamese high school EFL students and teachers (Table 10). The result shows that there was significant difference in perceptions towards D1 between Vietnamese high school EFL teachers $(\mathrm{M}=3.63, \mathrm{SD}=.79)$ and Vietnamese high school EFL students $(\mathrm{M}=3.47$, $\mathrm{SD}=.79)$ conditions; $\mathrm{t}(312)=1.98, \mathrm{p}=.049$. In addition, there was significant difference in perceptions towards D2 between Vietnamese high school EFL teachers $(\mathrm{M}=3.67, \mathrm{SD}=.71)$ and Vietnamese high school EFL students $(\mathrm{M}=3.84, \mathrm{SD}=.73)$ conditions; $\mathrm{t}(453)=-2.29, \mathrm{p}$ $=.023$. Lastly, there was significant difference in perceptions towards D3 between Vietnamese high school EFL teachers $(\mathrm{M}=4.20, \mathrm{SD}=.57)$ and Vietnamese high school EFL students $(\mathrm{M}$ $=4.08, \mathrm{SD}=.63)$ conditions; $\mathrm{t}(453)=2.05, \mathrm{p}=.041$.

\section{Table 10}

Independent samples t-test analysis of perceptions of Vietnamese high school EFL teachers and students towards ELF

\begin{tabular}{|c|c|c|c|c|c|c|}
\hline \multirow[t]{2}{*}{ Dimensions } & \multicolumn{2}{|c|}{$\begin{array}{l}\text { High school } \\
\text { teachers } \\
(\mathrm{N}=155)\end{array}$} & \multicolumn{2}{|c|}{$\begin{array}{l}\text { High school } \\
\text { students } \\
(\mathrm{N}=300)\end{array}$} & \multirow[t]{2}{*}{ t-value } & \multirow[t]{2}{*}{ Sig. } \\
\hline & Mean & SD & Mean & SD & & \\
\hline Overall & 3.86 & .43 & 3.82 & .52 & 1.07 & .287 \\
\hline D1 & 3.63 & .79 & 3.47 & .79 & 1.98 & $.049 *$ \\
\hline D2 & 3.67 & .71 & 3.84 & .73 & -2.29 & $.023^{*}$ \\
\hline D3 & 4.20 & .57 & 4.08 & .63 & 2.05 & $.041^{*}$ \\
\hline D4 & 3.74 & .75 & 3.79 & .93 & -.61 & .541 \\
\hline
\end{tabular}

An independent samples t-test analysis was conducted for items 1, 2, and 3 (Table 11). The result shows there was significant difference in Item 1 between Vietnamese high school EFL teachers $(\mathrm{M}=4.00, \mathrm{SD}=.94)$ and Vietnamese high school EFL students $(\mathrm{M}=3.73, \mathrm{SD}=1.04)$ conditions; $\mathrm{t}(453)=2.76, \mathrm{p}=.006$. 


\section{Table 11}

Independent samples t-test analysis of perceptions of Vietnamese high school EFL teachers and students towards models of English used in teaching materials

\begin{tabular}{|c|c|c|c|c|c|c|}
\hline \multirow[t]{2}{*}{ Items } & \multicolumn{2}{|c|}{$\begin{array}{l}\text { High school teachers } \\
\qquad(\mathrm{N}=155)\end{array}$} & \multicolumn{2}{|c|}{$\begin{array}{l}\text { High school students } \\
\qquad(\mathrm{N}=300)\end{array}$} & \multirow[b]{2}{*}{ t-value } & \multirow[b]{2}{*}{ Sig. } \\
\hline & Mean & $\mathrm{SD}$ & Mean & SD & & \\
\hline 1 & 4.00 & .94 & 3.73 & 1.04 & 2.76 & $.006^{* *}$ \\
\hline 2 & 3.72 & 1.09 & 3.51 & 1.11 & 1.92 & .055 \\
\hline 3 & 3.16 & 1.15 & 3.17 & 1.22 & -.10 & .92 \\
\hline
\end{tabular}

An independent samples $t$-test analysis was conducted for items 4 and 5 (Table 12). The result shows there was significant difference in Item 5 between Vietnamese high school EFL teachers $(\mathrm{M}=2.75, \mathrm{SD}=1.24)$ and Vietnamese high school EFL students $(\mathrm{M}=3.13, \mathrm{SD}=1.20)$ conditions; $\mathrm{t}(453)=-3.15, \mathrm{p}=.002$.

\section{Table 12}

Independent samples t-test analysis of perceptions of Vietnamese high school EFL teachers and students towards using English for communication

\begin{tabular}{|c|c|c|c|c|c|c|}
\hline \multirow[t]{2}{*}{ Items } & \multicolumn{2}{|c|}{$\begin{array}{l}\text { High school teachers } \\
\qquad(\mathrm{N}=155)\end{array}$} & \multicolumn{2}{|c|}{$\begin{array}{l}\text { High school students } \\
\qquad(\mathrm{N}=300)\end{array}$} & \multirow[b]{2}{*}{ t-value } & \multirow[b]{2}{*}{ Sig. } \\
\hline & Mean & $\mathrm{SD}$ & Mean & SD & & \\
\hline 4 & 4.59 & .61 & 4.54 & .69 & .77 & .443 \\
\hline 5 & 2.75 & 1.24 & 3.13 & 1.20 & -3.15 & $.002 * *$ \\
\hline
\end{tabular}

An independent samples $t$-test analysis was conducted for items 6, 7, 8, and 9. Table 13 presents the independent samples t-test analysis of perceptions of Vietnamese high school EFL teachers and students towards the role of language and culture in the English classroom. The result shows that there was significant difference in Item 8 between Vietnamese high school EFL teachers $(\mathrm{M}=4.06, \mathrm{SD}=.90)$ and Vietnamese high school EFL students $(\mathrm{M}=3.68, \mathrm{SD}=1.04)$ conditions; $\mathrm{t}(453)=3.89, \mathrm{p}=.000$.

\section{Table 13}

Independent samples t-test analysis of perceptions of Vietnamese high school EFL teachers and students towards the role of language and culture in the English classroom

\begin{tabular}{ccccccc}
\hline Items & $\begin{array}{c}\text { High school teachers } \\
(\mathrm{N}=155)\end{array}$ & \multicolumn{2}{c}{$\begin{array}{c}\text { High school students } \\
(\mathrm{N}=300)\end{array}$} \\
& Mean & SD & Mean & SD & t-value & Sig. \\
\hline 6 & 4.27 & .80 & 4.38 & .82 & -1.32 & .189 \\
7 & 4.39 & .62 & 4.29 & .79 & 1.53 & .126 \\
8 & 4.06 & .90 & 3.68 & 1.04 & 3.89 & $.000^{* * *}$ \\
9 & 4.07 & .84 & 3.95 & .92 & 1.37 & .172 \\
\hline
\end{tabular}

\section{A comparison between Vietnamese EFL teachers and students from gifted high schools.}

An independent samples $t$-test was conducted to compare perceptions towards English as a lingua franca between Vietnamese EFL students and teachers from gifted high schools (Table 14). The result shows that there was significant difference in perceptions towards ELF between Vietnamese EFL teachers from gifted high schools $(M=4.00, S D=.49)$ and Vietnamese gifted 
high school EFL students $(\mathrm{M}=3.80, \mathrm{SD}=.47)$ conditions; $\mathrm{t}(169)=-2.07, \mathrm{p}=.04$. Particularly, there was significant difference in perceptions towards D1 between Vietnamese high school EFL teachers $(\mathrm{M}=3.93, \mathrm{SD}=.88)$ and Vietnamese high school EFL students $(\mathrm{M}=3.46, \mathrm{SD}$ $=.78)$ conditions; $\mathrm{t}(169)=-2.94, \mathrm{p}=.004$.

\section{Table 14}

Independent samples t-test analysis of perceptions of Vietnamese high school EFL teachers and students from gifted high schools towards ELF

\begin{tabular}{|c|c|c|c|c|c|c|}
\hline \multirow[t]{2}{*}{ Dimensions } & \multicolumn{2}{|c|}{$\begin{array}{l}\text { Teachers from gifted high } \\
\text { schools } \\
(\mathrm{N}=30)\end{array}$} & \multicolumn{2}{|c|}{$\begin{array}{l}\text { Students from gifted high } \\
\text { schools } \\
(\mathrm{N}=141)\end{array}$} & \multirow{2}{*}{$\begin{array}{c}\mathrm{t}- \\
\text { value }\end{array}$} & \multirow[t]{2}{*}{ Sig. } \\
\hline & Mean & $\mathrm{SD}$ & Mean & $\mathrm{SD}$ & & \\
\hline Overall & 4.00 & .49 & 3.80 & .47 & -2.07 & $.04 *$ \\
\hline D1 & 3.93 & .88 & 3.46 & .78 & -2.94 & $.004 * *$ \\
\hline D2 & 3.73 & .81 & 3.74 & .69 & .03 & .98 \\
\hline D3 & 4.31 & .49 & 4.13 & .54 & -1.70 & .09 \\
\hline D4 & 3.75 & .80 & 3.73 & .92 & -.11 & .91 \\
\hline
\end{tabular}

An independent samples $t$-test analysis was conducted for all items (Table 15). The result shows there was significant difference in Item 1 between Vietnamese EFL teachers $(M=4.23, S D$ $=.94)$ and Vietnamese EFL students $(\mathrm{M}=3.80, \mathrm{SD}=1.02)$ from gifted high schools conditions; $\mathrm{t}(169)=-2.13, \mathrm{p}=.035$. Besides, the result shows there was significant difference in Item 3 between Vietnamese high school EFL teachers $(\mathrm{M}=3.63, \mathrm{SD}=1.10)$ and Vietnamese high school EFL students $(\mathrm{M}=3.06, \mathrm{SD}=1.16)$ conditions; $\mathrm{t}(169)=-2.50, \mathrm{p}=.013$. Similarly, there was significant difference in Item 8 between Vietnamese $E F L$ teachers $(M=4.40, S D$ $=.72)$ and Vietnamese EFL students $(\mathrm{M}=3.77, \mathrm{SD}=.92)$ from gifted high schools conditions; $\mathrm{t}(169)=-3.53, \mathrm{p}=.001$.

\section{Table 15}

Detailed independent samples t-test analysis of perceptions of Vietnamese high school EFL teachers and students from gifted high schools towards ELF

\begin{tabular}{ccccccc}
\hline Items & $\begin{array}{c}\text { Teachers from gifted high schools } \\
(\mathrm{N}=30)\end{array}$ & \multicolumn{2}{c}{$\begin{array}{c}\text { Students from gifted high schools } \\
(\mathrm{N}=141)\end{array}$} \\
& Mean & SD & Mean & SD & t-value & Sig. \\
\hline 1 & 4.23 & .94 & 3.80 & 1.02 & -2.13 & $.035^{*}$ \\
2 & 3.93 & 1.05 & 3.53 & 1.05 & -1.91 & .058 \\
3 & 3.63 & 1.10 & 3.06 & 1.16 & -2.50 & $.013^{*}$ \\
4 & 4.57 & .57 & 4.52 & .70 & -.36 & .72 \\
5 & 4.27 & .58 & 2.96 & 1.15 & .24 & .81 \\
6 & 4.27 & .83 & 4.39 & .79 & .77 & .44 \\
7 & 4.27 & .58 & 4.33 & .74 & .41 & .68 \\
8 & 4.40 & .72 & 3.77 & .92 & -3.50 & $.001^{* *}$ \\
9 & 4.30 & .75 & 4.01 & .90 & -1.62 & .107 \\
10 & 3.70 & .95 & 3.68 & 1.17 & -.10 & .92 \\
11 & 3.80 & 1.03 & 3.78 & 1.00 & -.10 & .92 \\
\hline
\end{tabular}




\section{A comparison between Vietnamese EFL teachers and students from non-gifted high schools.}

An independent samples $t$-test was conducted to compare perceptions towards ELF between Vietnamese EFL students and teachers from non-gifted high schools (Table 16). The result shows that there was significant difference in perceptions towards D2 between Vietnamese EFL teachers $(\mathrm{M}=3.66, \mathrm{SD}=.69)$ and Vietnamese EFL students $(\mathrm{M}=3.93, \mathrm{SD}=.76)$ from nongifted high schools conditions; $\mathrm{t}(282)=3.07, \mathrm{p}=.002$.

\section{Table 16}

Independent samples t-test analysis of perceptions of Vietnamese high school EFL teachers and students from non-gifted high schools towards ELF

\begin{tabular}{|c|c|c|c|c|c|c|c|}
\hline & \multirow[t]{2}{*}{ Dimensions } & \multicolumn{2}{|c|}{$\begin{array}{l}\text { Teachers from } \\
\text { non-gifted } \\
\text { high schools } \\
(\mathrm{N}=125)\end{array}$} & \multicolumn{2}{|c|}{$\begin{array}{c}\text { Students from } \\
\text { non-gifted high } \\
\text { schools } \\
(\mathrm{N}=159)\end{array}$} & \multirow[t]{2}{*}{$\mathrm{t}$-value } & \multirow[t]{2}{*}{ Sig. } \\
\hline & & Mean & SD & Mean & SD & & \\
\hline Overall & & 3.83 & .41 & 3.83 & .56 & .08 & .94 \\
\hline D1 & & 3.55 & .76 & 3.48 & .81 & .79 & .431 \\
\hline D2 & & 3.66 & .69 & 3.93 & .76 & -3.07 & $.002 * *$ \\
\hline D3 & & 4.17 & .59 & 4.03 & .70 & 1.86 & .064 \\
\hline D4 & & 3.74 & .75 & 3.84 & .95 & -1.03 & .303 \\
\hline
\end{tabular}

An independent samples $t$-test analysis was conducted for items 4 and 5. Table 17 presents the independent samples t-test analysis of perceptions of Vietnamese high school EFL teachers and students from gifted high schools towards using English for communication. The result shows that there was significant difference in Item 5 between Vietnamese EFL teachers $(M=2.72$, SD $=1.21)$ and Vietnamese EFL students $(\mathrm{M}=3.29, \mathrm{SD}=1.23)$ from non-gifted high schools conditions; $\mathrm{t}(282)=-3.89, \mathrm{p}=.000$.

\section{Table 17}

Independent samples t-test analysis of perceptions of Vietnamese high school EFL teachers and students from gifted high schools towards using English for communication

\begin{tabular}{|c|c|c|c|c|c|c|}
\hline \multirow[t]{2}{*}{ Items } & \multicolumn{2}{|c|}{$\begin{array}{c}\text { Teachers from non-gifted high } \\
\text { schools } \\
(\mathrm{N}=125)\end{array}$} & \multicolumn{2}{|c|}{$\begin{array}{l}\text { Students from non-gifted high } \\
\text { schools } \\
(\mathrm{N}=159)\end{array}$} & \multirow{2}{*}{$\begin{array}{c}\text { t- } \\
\text { value }\end{array}$} & \multirow[t]{2}{*}{ Sig. } \\
\hline & Mean & SD & Mean & SD & & \\
\hline 4 & 4.60 & .62 & 4.57 & .67 & .44 & .662 \\
\hline 5 & 2.72 & 1.21 & 3.29 & 1.23 & -3.89 & $.000 * * *$ \\
\hline
\end{tabular}

\section{Discussion}

\section{Summary of the Findings}

Table 18 presents the summary of the findings in relation to the three research questions. Firstly, Vietnamese high school EFL teachers positively perceived ELF. There were no significant differences between the perceptions of Vietnamese EFL teachers from gifted versus non-gifted 
high schools towards ELF in general and towards each of the dimensions, except D1 (Item 3). Secondly, Vietnamese high school EFL students positively perceived ELF. There were no significant differences between the perceptions of Vietnamese EFL students from gifted versus non-gifted high schools towards ELF in general and towards each of the dimensions, except D2 (Item 5). Finally, there were significant differences between the perceptions of Vietnamese EFL teachers and students from gifted high schools towards ELF (Items 1, 3, and 8) in general and towards D1 (Items 1, and 3) in particular. Besides, there was a significant difference between the perceptions of Vietnamese EFL teachers and students from non-gifted high schools towards D2 (Item 5). Also, there were substantial differences between the perceptions of Vietnamese high school EFL teachers and students towards D1 (Items 1), D2 (Item 5), and D3 (Item 8).

\section{Table 18}

Summary of the findings from quantitative data

\begin{tabular}{|c|c|c|c|}
\hline & Research Questions & \multicolumn{2}{|c|}{ Findings } \\
\hline \multirow[t]{2}{*}{1} & $\begin{array}{l}\text { How do Vietnamese high school EFL } \\
\text { teachers perceive ELF? }\end{array}$ & Positively & \\
\hline & $\begin{array}{l}\text { Are there any significant differences } \\
\text { between EFL teachers' perceptions } \\
\text { from gifted versus non-gifted high } \\
\text { schools towards ELF? }\end{array}$ & D1 (Item 3) & \\
\hline \multirow[t]{2}{*}{2} & $\begin{array}{l}\text { How do Vietnamese high school EFL } \\
\text { students perceive ELF? }\end{array}$ & Positively & \\
\hline & $\begin{array}{l}\text { Are there any significant differences } \\
\text { between the perceptions of gifted } \\
\text { versus non-gifted high school EFL } \\
\text { students towards ELF? }\end{array}$ & D2 (Item 5) & \\
\hline \multirow[t]{3}{*}{3} & $\begin{array}{l}\text { Are there any significant differences } \\
\text { between the perceptions of high school } \\
\text { EFL teachers and students towards }\end{array}$ & $\begin{array}{l}\text { High school teachers versus } \\
\text { students }\end{array}$ & $\begin{array}{l}\text { - D1 (Item 3) } \\
\text { - D2 (Item 5) } \\
\text { - D3(Item 8) }\end{array}$ \\
\hline & ELF? & $\begin{array}{l}\text { Teachers versus students } \\
\text { from gifted high schools }\end{array}$ & $\begin{array}{l}\text { - ELF (Items 1, 3, and 8) } \\
\text { - D1 (Items 1, and 3) }\end{array}$ \\
\hline & & $\begin{array}{l}\text { Teachers versus students } \\
\text { from non-gifted high schools }\end{array}$ & - D2 (Item 5) \\
\hline
\end{tabular}

\section{Models of English Used in Teaching Materials}

The results show that native and non-native English speakers' English models were considered necessary to be included in English teaching materials by both Vietnamese high school EFL teachers and students. Previous research shows similar conclusion (Curran \& Chern, 2017; Tran $\&$ Ngo, 2017). These relationships may partly be explained by the advent of the Internet (Tsai, 2004). More specifically, Zhang (2021) suggested using local English media and local topics to vary the accent options. This result may also be explained by the fact that English teacher training programs at universities have started to offer courses related to English varieties to raise pre-service teachers' awareness of this issue. Besides, Vietnamese teachers underwent pronunciation training, thus also being confident in their role as a good model for students 
(Quoc et al., 2021).

However, in this study's findings, models of native English speakers are still preferred by most. This is in accordance with the findings from the study on students and teachers at the tertiary level (Do, 2000; Ton \& Pham, 2010; Tran \& Moore, 2015). There are two possible explanations for this result. Firstly, paper-pencil exams, which are employed mostly all over the world, promote the use of certain native English models. Secondly, parents and society expect students to possess a native-like accent puts much pressure on students' efforts to imitate native English speakers. The influence of people in the broader public on the dominant use of native English models was also reported by Wang and Fang (2018).

One interesting finding is that there was a statistically significant difference in perceptions towards D1 between sub-groups of participants. Teachers and students working with more advanced English tend to support English varieties more strongly. However, the findings of the current study do not reinforce previous research. English minors who tended to work in business environments favored various models of English more than interns and English majors, most of whom would work in academia (Curran \& Chern, 2017). A possible explanation for this might be because participants in Curran and Chern's study (2017) were from Taipei, a densely populated city with multi-national inhabitants, while the respondents in the current study who could hardly have any chance to communicate with foreigners in English regularly were mostly from a homogeneous small province in Vietnam. Thus, the personal experiences of using English led to the diversity in perceptions towards ELF (Sung, 2017).

\section{Using English for Communication}

The current findings show that Vietnamese EFL teachers and students agreed on the ultimate purpose of learning English, which is communication. This result is in line with that from the research on Taiwanese pre-service teachers conducted by Curran and Chern (2017). Also, the purpose of using English to make friends with people all over the world was highly rated in the study by Tran et al. (2021). As stated above, a possible explanation for this might be the advances of modern technology, especially the Internet. However, in the current study, the idea of real-life communication seemed to be between Vietnamese students and native English speakers mainly. This difference can be partly explained by different living and working environments and the unawareness of the number of people speaking English classified based on Kachru's circle (1985). The Vietnamese participants had few chances to communicate with foreigners in English, and they did not even know that there were more non-native English speakers than native ones.

Notably, there was a statistically significant difference in perceptions towards using English for communication between Vietnamese gifted versus non-gifted high school EFL students, between Vietnamese EFL teachers and students from non-gifted high schools, and between Vietnamese high school EFL teachers and students with the higher mean scores rated by Vietnamese non-gifted high school EFL students, Vietnamese EFL students from non-gifted high schools, and Vietnamese high school students respectively. Since this difference has not 
been found elsewhere, the reason for this is not clear but it may have something to do with students' level of English and intrinsic motivation related to future jobs. Not like non-gifted students who study general English and want to focus on communication, gifted students have to study more advanced English; hence, for them, the purposes of learning English are both for communication and for academic use. Also, compared with teachers who expect their students to perform well in academic exams, students are more open to the purpose of learning English for communication with the hope of getting good jobs at international companies in the future. Lastly, the fact that Vietnamese society considers a student as an excellent one only if he/ she can communicate fluently in English in real life, not just high scores in exams, can also be another explanation for this finding.

\section{The Role of Language and Culture in the English Classroom}

The present study's findings show that cultures taught in English classrooms are no longer only those of native English speakers. Instead, Vietnamese EFL high school teachers and students believed that other cultures should also be equally introduced in the classroom so that students can communicate interculturally effectively. This finding is supported by Jenkins (2007) and Baker (2011). There are two possible explanations for this result. The first one is the change in the new textbooks that spare the last class period of each learning unit for culture. The second one is the diverse sources of teaching materials presenting different interesting cultures throughout the world.

Additionally, a statistically significant difference in perceptions towards the role of language and culture in the English classroom between Vietnamese high school EFL teachers and students with the higher mean scores rated by Vietnamese high school EFL teachers was found in the present study. It is difficult to explain this result, but it might be related to the habit of learning English from previous textbooks. It means that while teachers are positively changing their perceptions towards this issue owing to recent new-textbook training workshops, students have not been familiar with this new trend.

\section{Language Use in Classroom}

The current study's findings also show that a mixture of Vietnamese and English was strongly supported to be used in English classrooms to develop students' learning English to the best of its effect by both Vietnamese teachers and students. Effective code-switching strategies were also regarded as important. These results reflect those of researchers who claimed that compared to native English speakers, non-native English teachers whose first language is the same as their students would be more capable of assisting students in enhancing their English language skills (Curran \& Chern, 2017). These results are likely to be related to teachers' ability, students' level of English, and mixed-level classes. In reality, some teachers are not confident in their English-speaking ability; therefore, they rarely use English to instruct students. For classes with a large number of low achieving students, it seems impossible for teachers to use any English to instruct them. Besides, for mixed-level classes, using English in the classroom may consume much time since teachers have to use their mother tongue to explain again for 
low-achieving students. However, using English entirely motivates both students and teachers to self-study for English gifted classes.

\section{Conclusion}

The purpose of the study is to investigate how Vietnamese EFL students and teachers from gifted versus non-gifted high schools perceive four dimensions of English of a lingua franca, namely models of English use in teaching materials, using English for communication, the role of language and culture in English classroom, and language use in English classroom. The result shows that both Vietnamese EFL teachers and students bore positive perceptions towards all four dimensions of English as a lingua franca. Besides, the researcher also identified the differences among five groups: between Vietnamese EFL teachers from gifted and non-gifted high schools (difference in perceptions towards models of English used in teaching materials), between Vietnamese EFL high school gifted and non-gifted students (difference in perceptions towards using English for communication), between Vietnamese EFL teachers and students (difference in perceptions towards models of English used in teaching materials, using English for communication and the role of language and culture in the English classroom), between Vietnamese EFL teachers and students from gifted high schools (difference in perceptions towards models of English used in teaching materials and ELF in general), between Vietnamese EFL teachers and students from non-gifted high schools (difference in perceptions towards using English for communication).

Based on the present research findings, several theoretical and pedagogical implications for research, policymakers, Vietnamese high school EFL teachers, and learners were generated and discussed.

For research, the authors suggest that more research should target high-school students and inservice teachers. A cross-cultural study on the discussed issue should also be conducted to obtain a deeper understanding of factors influencing perceptions towards ELF.

For policymakers, the field of ELT materials design and evaluation needs to take findings from empirical research within the ELF scholarship into account. Also, teaching and learning English at the high school level are not only English for communication but also academic English because this is the preparation phase for students who might keep studying higher or leave school and work. Consequently, teaching English at high school should fulfill the dual-task at the foundation level. Additionally, raising awareness of the existence of ELF for both Vietnamese high school teachers is essential because of the vital role of teachers in shortening the gaps between ELF theories and practices (Yu \& Liu, 2021).

For Vietnamese high school EFL teachers, non-native English models are advised to be introduced briefly. To deal with the lack of an environment for practicing English, intrinsic motivation should be fostered when extrinsic motivation is not beneficial. Providing a vision of studying abroad, getting a high salary, or promoting to important positions in international 
companies can be effective. Besides, the use of technology to bring the world into the classroom via Skype, Zoom, or Microsoft Teams can help create good conditions for students to practice using ELF in the new learning environment. Similarly, assigning high-achieving students as group leaders in group discussions could help low-achieving ones have more chances to speak and lower the classroom management workload for teachers. Another considerable feature is that the culture lessons in textbooks primarily provide students with the questions of 'what' and 'how', so it is the teacher's responsibility to guide students to answer the question of 'why' so that learners could become culturally sensitive global citizens.

Finally, for Vietnamese high school EFL students, students should take advantage of different sources from the Internet, including those by native English speakers and non-native ones.

The most notable limitation of this study is that the Vietnamese EFL high school teachers and students were mostly from Southern Vietnam, particularly in Khanh Hoa province. Therefore, this is not a representative sample, thus cannot be generalized for the perceptions of all Vietnamese EFL high school teachers and students. In addition, the concept of ELF is certainly composed of many more complex dimensions than just four studied ones in the current study. Also, the limited number of gifted high schools may influence the mean comparisons between groups. Last but not least, the questionnaire originally used in the Taiwanese context was modified to contain only eleven items so that it could be used in the Vietnamese context, which may need to be tested in other contexts in order to enhance the reliability of the instrument.

\section{Acknowledgments}

This is a part of Nguyen Thien Bao's master thesis at Department of Applied Foreign Languages, NTUST, Taiwan under the supervision of Dr. Yi-Hsuan Gloria Lo.

\section{References}

Ambele, E. A., \& Boonsuk, Y. (2020). Voices of learners in Thai ELT classrooms: A wake-up call towards teaching English as a lingua franca. Asian Englishes. DOI: $\underline{10.1080 / 13488678.2020 .1759248}$

Baker, W. (2011). Global cultures and identities: refocusing the aims of ELT in Asia through intercultural awareness. In Muller, T., Herder, S., Adamson, J. and Brown, P.S. (eds), Innovating EFL Education in Asia, 23-34. Basingstoke: Palgrave Macmillan.

Butler, Y. G., Lee, J., \& Peng, X. (2020). Failed policy attempts for measuring English speaking abilities in college entrance exams. English Today, First View, 1-7.

Curran, J. E., \& Chern, C. L. (2017). Pre-service English teachers' attitudes towards English as a lingua franca. Teaching and Teacher Education, 66, 137-146. DOI: 10.1016/j.tate.2017.04.007

Decision 82 (2008). Article 3. Ministry of Education and Training. Retrieved from 
https://www.elib.vn/quyet-dinh-82-2008-qd-bgddt-quy-che-to-chuc-va-hoat-dong-cuatruong-trung-hoc-pho-thong-chuyen-447808.html

DeVellis, R. F. (2012). Scale development: Theory and applications (3rd ed.). Thousand Oaks, CA: SAGE.

Dewey, M. (2012). Towards a post-normative approach: learning the pedagogy of ELF. Journal of English as a Lingua Franca, 1(1), 141-170. DOI: 10.1515/jelf-2012-0007

Do, H. T. (2000). Foreign language education in Vietnam: The emergence of English and its impact on higher education. In J. Shaw, D. Lubeska, \& M. Noullet (Eds.), Language and development: Partnership and interaction: Proceedings of the Fourth International Conference on Language and Development. Bangkok: Asian Institute of Technology.

Guohai, L. \& Geling, H. (2021). English as a lingua franca: The pragmatic perspective. International Journal of Bilingual Education and Bilingualism. DOI: $\underline{10.1017 / 9781316217832}$

Haberland, H. (2011). Ownership and maintenance of a language in transnational use: Should we leave our lingua franca alone?. Journal of Pragmatics, 43, 937-949. DOI: $\underline{10.1016 / j . p r a g m a .2010 .08 .009}$

Ho, M. H. T., \& Nguyen, H. T. (2019). English as a lingua franca for Vietnam. In L., V., Canh, H. T. M. Nguyen \& R., Barnard (Ed.). Building teacher capacity in English language teaching in Vietnam: Research, Policy and Practice (pp. 166-183). London: Routledge.

Jenkins, J. (2000). The phonology of English as an international language. Oxford: Oxford University Press.

Jenkins, J. (2007). English as a lingua franca: Attitude and identity. Oxford: Oxford University Press.

Jenkins, J. (2012). English as a Lingua Franca from the classroom to the classroom. ELT Journal, 66(4), 486-494. DOI: $10.1093 / \mathrm{elt} / \mathrm{ccs} 040$

Kachru, B. B. (1985). World Englishes and applied linguistics. World Englishes, 9, 3-20.

Kim, S. (2021). English as a lingua franca in Japan: Multilingual postgraduate students' attitudes towards English accents. Journal of Multilingual and Multicultural Development. DOI: $10.1080 / 01434632.2021 .1909053$

Kontra, E., \& Csizer, K. (2011). "They can achieve their aims without native skills in the field of work or studies": Hungarian students' views on English as a lingua franca. Studies in Second Language Learning and Teaching, 1(1), 135-152. DOI: 10.14746/ssllt.2011.1.1.8

Laitinen, M. (2020). Empirical perspectives on English as a lingua franca (ELF) grammar. World Englishes, 39(3), 427-442. DOI: 10.1111/weng.12482

Ministry of Education of Vietnam. (2018). Important missions in 2018 and 2019 - National languages project from 2017 to 2025. Retrieved from 
http://ngoainguquocgia.moet.gov.vn/Portals/Legals/636688615896193821so $\% 203258 \% 202.8 \% 20$ Hoan $\% 20$ thien $\% 20$ KH\%20giai\%20doan $\% 202017$ 2025\%20va $\% 20$ huong\%20dan $\% 20$ nhiem $\% 20$ vu $\% 20$ trong $\% 20$ tam $\% 20$ nam $\% 202018 \%$ 20va\%20nam $\% 202019 \% 20$ cua $\% 20$ DANN.pdf.

Nguyen, H. T. (2019). Developing interactional competence in a lingua franca at the workplace: An ethnomethodologically endogenous account. In H. Nguyen \& T. Malabarba (Ed.), Conversation Analytic Perspectives on English Language Learning, Teaching and Testing in Global Contexts (pp. 59-84). Bristol, Blue Ridge Summit: Multilingual Matters.

Nguyen, T. M. P., Jin, P., \& Gross, M. U. M. (2013). Confucian values in Vietnamese gifted adolescents and their non-gifted peers. Gifted and Talented International, 28, 227-238. DOI: $\underline{10.1080 / 15332276.2013 .11678417}$

Nguyen, V. L., \& Ngo, Q. M. H. (2018). English as a medium of instruction: A case study at a gifted high school in Vietnam. The Journal of Asia TEFL, 15 (4), 1083-1102. DOI: 10.18823/asiatefl.2018.15.4.13.1083

Osimk-Teasdale, R. (2015). Parts of speech in English as a lingua franca: the POS tagging of VOICE. Unpublished PhD thesis. University of Vienna.

Pang, Y. (2020). The cognitive saliency of word associations of verbs of speech in English as a lingua franca interactions. Intercultural Pragmatics, 17(4), 417-443. DOI: $10.1515 / \mathrm{ip}-$ 2020-4002

Phan, H. L. T. (2020). Vietnamese learners' attitudes towards American and British accents. European Journal of English Language Teaching, 6(2), 97-117. DOI: $\underline{10.46827 / \text { jel.v6i2.3498 }}$

Quoc, T. X., Thanh, V. Q., Dang, T. D. M., Mai, N. D. N., \& Nguyen, P. N. K. (2021). Teachers' perspectives and practices in teaching English pronunciation at Menglish center. International Journal of TESOL \& Education, 1(2), 158-175. DOI: http://eoi.citefactor.org/10.11250/ijte.01.02.009

Ruth, O. T. (2018). Analyzing ELF variability. In Jenkins, J., Baker, W., \& Dewey, M. The Routledge handbook of English as a lingua franca. London and New York: Routledge.

Seidlhofer, B. (2001). Closing a conceptual gap: The case for a description of English as a Lingua Franca. International Journal of Applied Linguistics, 11(2), 133-158. DOI: $\underline{10.1111 / 1473-4192.00011}$

Shohamy, E., \& Hornberger, N. H. (2017). Language testing and assessment. Encyclopedia of language and education.

Sonderen, E. V., Sanderman, R., Coyne, J. C. (2013). Ineffectiveness of reverse wording of questionnaire items: Let's learn from cows in the rain. PLoS ONE, 8(7), e68967.

Sung, C. C. M. (2017). Investigating perceptions of English as a lingua franca in Hong Kong: The case of university students. English Today, 34(1), 38-44. DOI: 


\section{$\underline{10.1017 / \mathrm{S} 0266078417000293}$}

Ton, N.N.H, \& Pham, H.H (2010). Vietnamese teachers' and students' perceptions of global English. Language Education in Asia, 1(1), 48-61.

Tran, T. H., \& Moore, P. (2015). Vietnamese English teachers' perceptions on incorporating World Englishes into their teaching. Language Education in Asia, 6(2), 107-121.

Tran, T. Q. T., Nguyen, T. M. N., Luu, T. T., \& Pham, T. B. N. (2021). An evaluation of English non-majored freshmen's attitude towards EFL learning at Can Tho University of Technology. International Journal of TESOL \& Education, 1(2), 72-98. Retrieved from http://i-jte.org/index.php/journal/article/view/35

Tsai, C. C. (2004). Beyond cognitive and metacognitive tools: The use of the Internet as an 'epistemological' tool for instruction. British Journal of Educational Technology, 35, 525536.

Víctor, P. G., \& Lanteigne, B. (2020). Morpho-syntactic features of English as a lingua franca in Dubai and Sharjah. In P. Siemund \& J. r. E. Leimgruber (ed.), Multilingual Global Cities: Singapore, Hong Kong, Dubai (pp. 303-320). London: Routledge.

Vietnamese educational law (2005). Article 62. Retrieved from https:/www.wto.org/english/thewto e/acc e/vnm e/WTACCVNM43 LEG 14.pdf

$\mathrm{Vu}, \mathrm{P}$. (2011). Gifted students profiles and their attitudes towards a gifted program: The case of Vietnam. Gifted and Talented International, 26, 81-87.

$\mathrm{Vu}, \mathrm{P}$., Vu, L. (2012). Teaching English as a Foreign Language Major to Gifted Students. The Southeast Asian Journal of English Language Studies, 18 (2), 57-66.

Wang, W., \& Fang, F. (2018). Chinese netizens' reactions to the use of English as a lingua franca. English Today, 35(4), 3-12. DOI: https://doi.org/10.1017/S0266078418000482

Widdowson, H.G. (2012). ELF and the inconvenience of established concepts. Journal of English as a Lingua Franca, 1(1), 5-26.

Widdowson, H.G. (2015). ELF and the pragmatics of language variation. Journal of English as a Lingua Franca, 4(2), 359-372.

Wong, N., Rindfleisch, A., \& Burroughs, J. E. (2003). Do reverse-worded items confound measures in cross-cultural consumer research? The case of the material values scale. Journal of Consumer Research, 30, 72 - 91. DOI: 10.1086/374697

Wu, X., Mauranen, A., \& Lei, L. (2020). Syntactic complexity in English as a lingua franca academic writing. Journal of English for Academic Purposes, 43, 100798. DOI: $\underline{10.1016 / j . j e a p .2019 .100798}$

Yalçın, Ş., Bayyurt, Y., \& Alahdab, B. R. (2020). Triggering effect of CLIL practice on English as a lingua franca awareness. ELT Journal, 74(4), 387-397. 
Yao, C. W., Garcia, C. E., \& Collins, C. (2019). English as lingua franca: Exploring the challenges and opportunities of English language on Vietnamese graduate student learning. Journal for the Study of Postsecondary and Tertiary Education, 4, 209-225. DOI: $\underline{10.28945 / 4391}$

Yu, X., \& Liu, C. Y. (2021). Teaching English as a lingua franca in China. English Today, First View, 1-9. DOI: https://doi.org/10.1017/S0266078421000018

Zhang, Q. (2021). Impacts of World Englishes on local standardized language proficiency testing in the Expanding Circle. English Today, First View, 1-17. DOI: $\underline{10.1017 / \mathrm{S} 0266078421000158}$

\section{Biodata}

Nguyen Thien Bao is currently a doctoral student in Linguistics at National Chung Cheng University, Taiwan. He received his first master's degree in Applied Foreign Languages, and his second master's degree in Educational Leadership and Management Development. His research interests include Educational Linguistics, and Educational Technology.

Yi-Hsuan Gloria Lo is currently a professor \& the Chair of the Department of Applied Foreign Languages at NTUST, Taipei, Taiwan. She received her PhD in Language Education from Indiana University, Bloomington, USA. Her research areas include Sociocultural Perspectives of Teaching and Learning, Curriculum Design, and Professional Development of English Teachers. 


\section{APPENDIX 1}

The newly modified scale

$(1=$ strongly disagree, $2=$ disagree, $3=$ neutral, $4=$ agree, $5=$ strongly agree $)$

\begin{tabular}{|c|c|c|c|c|c|c|}
\hline Items & Statements & & 5-pc & & & \\
\hline 1 & $\begin{array}{l}\text { I think it is important that students be exposed to English spoken by a } \\
\text { range of native speakers (e.g, British English, Australian English, } \\
\text { American English, South African English...) }\end{array}$ & 1 & 2 & 3 & 4 & 5 \\
\hline 2 & $\begin{array}{l}\text { I think it is important that students be exposed to English used by } \\
\text { proficient second-language speakers. }\end{array}$ & 1 & 2 & 3 & 4 & 5 \\
\hline 3 & $\begin{array}{l}\text { I think it is important that classroom materials provide a range of } \\
\text { models of English used by L1 and L } 2 \text { speakers. }\end{array}$ & 1 & 2 & 3 & 4 & 5 \\
\hline 4 & $\begin{array}{l}\text { I think the major focus of an English program should be teaching } \\
\text { students to use the language in real-life communication. }\end{array}$ & 1 & 2 & 3 & 4 & 5 \\
\hline 5 & $\begin{array}{l}\text { I think an important focus of an English program should be to prepare } \\
\text { students for communication with people who are not English native } \\
\text { speakers. }\end{array}$ & 1 & 2 & 3 & 4 & 5 \\
\hline 6 & $\begin{array}{l}\text { I think it is important that students can use English to share information } \\
\text { about their own culture and traditions. }\end{array}$ & 1 & 2 & 3 & 4 & 5 \\
\hline 7 & $\begin{array}{l}\text { I think it is important that English teachers help students to better } \\
\text { understand exchange students or people from other countries with } \\
\text { whom they are likely to use English. }\end{array}$ & 1 & 2 & 3 & 4 & 5 \\
\hline 8 & $\begin{array}{l}\text { I think it is important that students become familiar with the cultures } \\
\text { and traditions of speakers of English (both native and non-native ones). }\end{array}$ & 1 & 2 & 3 & 4 & 5 \\
\hline 9 & $\begin{array}{l}\text { I think it is important to teach students to be aware of intercultural } \\
\text { differences and encourage them to talk about such differences. }\end{array}$ & 1 & 2 & 3 & 4 & 5 \\
\hline 10 & $\begin{array}{l}\text { I think a bilingual approach where Vietnamese is used as a support in } \\
\text { English language classes is more effective for Vietnamese students. }\end{array}$ & 1 & 2 & 3 & 4 & 5 \\
\hline 11 & $\begin{array}{l}\text { I think it is important to teach students strategies of effective code- } \\
\text { switching between English and Vietnamese. }\end{array}$ & 1 & 2 & 3 & 4 & 5 \\
\hline
\end{tabular}

